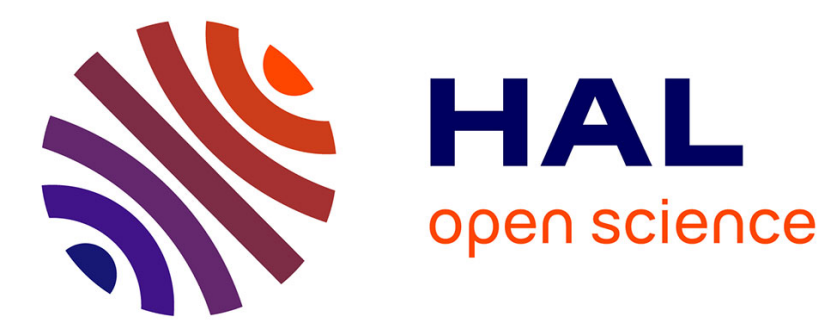

\title{
Orthogonal polynomial sets with finite codimensions
}

Bertrand G. Giraud, M.L. Mehta, A. Weiguny

\section{To cite this version:}

Bertrand G. Giraud, M.L. Mehta, A. Weiguny. Orthogonal polynomial sets with finite codimensions. Comptes Rendus. Physique, 2004, 5 (7), pp.781-787. 10.1016/j.crhy.2004.09.017 . cea-02904716

\section{HAL Id: cea-02904716 https://hal-cea.archives-ouvertes.fr/cea-02904716}

Submitted on 9 Nov 2020

HAL is a multi-disciplinary open access archive for the deposit and dissemination of scientific research documents, whether they are published or not. The documents may come from teaching and research institutions in France or abroad, or from public or private research centers.
L'archive ouverte pluridisciplinaire HAL, est destinée au dépôt et à la diffusion de documents scientifiques de niveau recherche, publiés ou non, émanant des établissements d'enseignement et de recherche français ou étrangers, des laboratoires publics ou privés. 


\title{
Orthogonal polynomial sets with finite codimensions
}

\author{
Bertrand G. Giraud ${ }^{a}$, M.L. Mehta ${ }^{a}$, A. Weiguny ${ }^{b}$ \\ a Service de physique théorique, CNRS, CE Saclay, 91191 Gif/Yvette, France \\ ${ }^{\mathrm{b}}$ Institut für Theoretische Physik, Univeristät Münster, Germany
}

Received 15 April 2004; accepted after revision 20 September 2004

Presented by Roger Balian

\begin{abstract}
We define sets of orthogonal polynomials which lack one or several degrees, because of a finite number of constraints. In particular, we are interested in a generalization of Hermite polynomials, governed by a constraint of zero average. These are of interest, for example, for the study of the Hohenberg-Kohn functional. In particular, they allow the calculation of potential perturbations which generate strictly proportional density perturbations. To cite this article: B.G. Giraud et al., C. R. Physique 5 (2004).

(C) 2004 Académie des sciences. Published by Elsevier SAS. All rights reserved.
\end{abstract}

\section{Résumé}

Polynômes orthogonaux contraints. Nous définissons des ensembles des polynômes orthogonaux sous des contraintes diverses. C'est notamment les cas d'une généralisation de polynômes d'Hermite, contraintes par une moyenne de zéro, pour la fonctionnelle de Hohenberg-Kohn. Ils permettent le calcul des perturbations de potentiel qui engendrent strictement la même forme pour les perturbations de densité. Pour citer cet article: B.G. Giraud et al., C. R. Physique 5 (2004).

(ㄷ) 2004 Académie des sciences. Published by Elsevier SAS. All rights reserved.

\section{Version française abrégée}

Dans de nombreux problèmes de physique mathématique il est nécessaire de considérer des développements sur des bases de fonctions satisfaisant à des contraintes. C'est notamment le cas de la fonctionnelle de Hohenberg-Kohn [1], d'usage fréquent en physique atomique, moléculaire et nucléaire. En effet elle lie de façon biunivoque la densité $\rho(r)$ d'un système et son potentiel à un corps $u(r)$, sous deux conditions : (a) $\int \delta \rho(r)=0$, pour la conservation du nombre de particules; et (b) $\delta u(r) \neq c t e$, pour une variation non triviale du potentiel. Ces contraintes permettent de calculer correctement les dérivées fonctionnelles $\delta u / \delta \rho$ et $\delta \rho / \delta u$. Dans cette note, nous définissons des bases de fonctions $w_{n}(r)=e^{-r^{2} / 2} \Gamma_{n}(r)$, où les $\Gamma_{n}(r)$ sont, sous des contraintes diverses, des polynômes orthogonaux par rapport à la mesure $e^{-r^{2}} \mathrm{~d} r$ de support ] $-\infty, \infty[$. Ce sont donc des modifications de polynômes d'Hermite. Un premier ensemble de polynômes est défini selon les Eqs. (1) par la contrainte $\int_{-\infty}^{\infty} e^{-r^{2} / 2} A_{n}(r) \mathrm{d} r=0$. Le polynôme constant $A_{0}$ est donc incompatible avec la contrainte. La forme explicite des $A_{n}$ compatibles est donnée par les Eqs. (3), ainsi qu'une relation de récurrence par l'Eq. (7). Une autre contrainte,

E-mail addresses: giraud@spht.saclay.cea.fr (B.G. Giraud), mehta@spht.saclay.cea.fr (M.L. Mehta), weiguny@uni-muenster.de (A. Weiguny). 
$\int_{-\infty}^{\infty} e^{-r^{2} / 2} r B_{n}(r) \mathrm{d} r=0$, voir les Eqs. (17), élimine le polynôme $B_{1}$ et définit un autre ensemble de polynômes, dont la formule explicite est donnée par les Eqs. (18). Une première application pratique de ces polynômes est proposée : ils permettent de calculer des perturbations de potentiel qui engendrent strictement la même forme pour les perturbations de densité.

\section{Introduction}

Polynomials which are orthogonal but do not form a complete set may be important for some applications. They do not belong to the two kinds of multiple orthogonal polynomials studied by van Assche [2]. Rather, we design them according to constraints. In particular, the present study was prompted by the consideration of the Hohenberg-Kohn functional and similar functionals along the Thomas-Fermi method [1,3,4], of a general use in atomic, molecular and nuclear theoretical physics. Indeed, in such functional approaches, the ground state energy of a system is a functional of its density $\rho(r)$, and there is a special interest in the mean field $u(r)$ driving the system. It is thus convenient to expand variations of $\rho$ in a basis $\left\{w_{m}(r)\right\}$ of particle number conserving components, $\delta \rho(r)=\sum_{m} \delta \rho_{m} w_{m}(r)$, with the constraint, $\forall m, \int \mathrm{d} r w_{m}(r)=0$. Simultaneously, it is convenient to expand variations of $u$ in a basis orthogonal to the flat potential, because, trivially, a flat $u$, as just a change in energy references, cannot influence the density. The same basis can thus be used for $\delta u(r)=\sum_{n} \delta u_{n} w_{n}(r)$, since the very same condition, $\int \mathrm{d} r w_{n}(r)=0$, expresses orthogonality to a constant $u$.

We will consider two examples, missing the first or the second polynomial in the normal degree hierarchy running from $m=0$ to $\infty$. These are the subjects of Sections 2 and 3. A numerical, illustrative example, shows how to define 'eigen-densitypotential modes' where potential perturbations generate density perturbations which have exactly proportional forms. A brief discussion and conclusion make Section 5.

\section{Hermite polynomials under a constraint of zero average}

In this section we consider basis states carrying a weight $e^{-r^{2} / 2}$, in the form $w_{n}(r)=e^{-r^{2} / 2} A_{n}(r)$, where $A_{n}$ is a polynomial. It is clear that $A_{0}$ cannot be a finite, non-vanishing constant if the constraint, $\int \mathrm{d} r e^{-r^{2} / 2} A_{0}(r)=0$, must be implemented. Hence let polynomials $A_{n}(r)$ of precise order $n, n \geqslant 1$, be defined by,

$$
\int_{-\infty}^{\infty} e^{-r^{2}} A_{m}(r) A_{n}(r) \mathrm{d} r=a_{n} \delta_{m n}, \quad m \geqslant 1, n \geqslant 1, \quad \int_{-\infty}^{\infty} e^{-\frac{1}{2} r^{2}} A_{m}(r) \mathrm{d} r=0, \quad m \geqslant 1,
$$

where the $a_{n}$ are normalizations, defined later. The constant polynomial $A_{0}(r)$ is left out of the orthogonality relations. From a trivial parity argument one sees that the odd (indexed) polynomials, undisturbed by leaving out $A_{0}$, will be proportional to the Hermite polynomials. Only the even (indexed) polynomials are modified. In fact, with $H_{n}(r)$ the usual Hermite polynomials,

$$
H_{n}(r)=e^{r^{2}}\left(-\frac{\mathrm{d}}{\mathrm{d} r}\right)^{n} e^{-r^{2}}=n ! \sum_{j=0}^{[n / 2]}(-1)^{j} \frac{(2 r)^{n-2 j}}{j !(n-2 j) !},
$$

one can verify that

$$
A_{2 n+1}(r)=H_{2 n+1}(r), \quad A_{2 n}(r)=H_{2 n}(r)-(n-1) ! 2^{2 n-1} \sum_{j=0}^{n-1} \frac{H_{2 j}(r)}{2^{2 j} j !},
$$

satisfy Eqs. (1) for $n, m \geqslant 1$ with

$$
a_{2 n}=2^{2 n}(2 n) !\left(1+\frac{1}{2 n}\right) \sqrt{\pi}, \quad a_{2 n+1}=2^{2 n+1}(2 n+1) ! \sqrt{\pi} .
$$

For this verification one needs

$$
\int_{-\infty}^{\infty} e^{-r^{2}} H_{n}^{2}(r) \mathrm{d} r=2^{n} n ! \sqrt{\pi}, \quad \int_{-\infty}^{\infty} e^{-\frac{1}{2} r^{2}} H_{2 n}(r) \mathrm{d} r=\frac{(2 n) !}{n !} \sqrt{2 \pi}, \quad \sum_{j=0}^{n} \frac{(2 j) !}{2^{2 j}(j !)^{2}}=\frac{(2 n+1) !}{2^{2 n}(n !)^{2}} .
$$

The last equation is a special case of the more general identity

$$
\sum_{j=0}^{n} \frac{\Gamma(j+a)}{\Gamma(j+1)}=\frac{\Gamma(n+a+1)}{a \Gamma(n+1)}
$$


for $a=1 / 2$ and can be proven by induction.

The polynomials $A_{n}(x)$ obey the following three term recursion (differential-difference) relation

$$
A_{n+1}(r)=2 r A_{n}(r)-2 A_{n}^{\prime}(r)+2\left[n+(-1)^{n}\right] A_{n-1}(r),
$$

where a prime denotes the derivative. It is straightforward to verify this by substituting Eqs. (3) for $A_{n}$ and using

$$
2 r H_{n}(r)=H_{n+1}(r)+H_{n}^{\prime}(r)=H_{n+1}(r)+2 n H_{n-1}(r),
$$

or one may argue as follows:

1. By partial integration,

$$
\int_{-\infty}^{\infty} e^{-\frac{1}{2} r^{2}} A_{n}^{\prime}(r) \mathrm{d} r=\int_{-\infty}^{\infty} e^{-\frac{1}{2} r^{2}} r A_{n}(r) \mathrm{d} r
$$

so that $\left(r A_{n}-A_{n}^{\prime}\right)$ can be expressed as a linear combination of the $A_{k}(r)$ with $n+1 \geqslant k>0$. One does not need $A_{0}$.

2. The coefficient of the $k$ th term obtains by an integration of $\left[r A_{n}(r)-A_{n}^{\prime}(r)\right]$ against $A_{k}(r)$, weighted by $e^{-r^{2}}$. But, by partial integration of the $A_{n}^{\prime}$ term, one obtains, if $k<n-1$,

$$
\int_{-\infty}^{\infty} e^{-r^{2}}\left[r A_{n}(r)-A_{n}^{\prime}(r)\right] A_{k}(r) \mathrm{d} r=\int_{-\infty}^{\infty} e^{-r^{2}} A_{n}(r)\left[A_{k}^{\prime}(r)-r A_{k}(r)\right] \mathrm{d} r=0,
$$

by the usual argument, since $\left[r A_{k}(r)-A_{k}^{\prime}(r)\right]$ is then a polynomial expressible as a linear combination of the $A_{j}(r)$ with $0<j \leqslant k+1<n$. Furthermore, since $A_{n}$ does not have the correct parity to contribute to the expansion of $\left(r A_{n}-A_{n}^{\prime}\right)$, only $A_{n+1}$ and $A_{n-1}$ are left.

3. Coefficients of $A_{n+1}(r)$ and $A_{n-1}(r)$ can be fixed by computing explicitly the coefficients of the powers $r^{n+1}$ and $r^{n-1}$ or by the use of the orthogonality relation. If the normalizations are set so that the highest degree term of $A_{n}$ is $(2 r)^{n}$, a condition fulfilled by the Hermite polynomials and by the ansatz, Eqs. (3), then it is consistent to set

$$
r A_{n}(r)-A_{n}^{\prime}(r)=\frac{1}{2} A_{n+1}(r)+\alpha_{n-1} A_{n-1}(r) .
$$

Again a partial integration of the $A_{n}^{\prime}$ term then gives

$$
\int e^{-r^{2}}\left[r A_{n}-A_{n}^{\prime}\right] A_{n-1} \mathrm{~d} r=\int e^{-r^{2}} A_{n}\left[A_{n-1}^{\prime}-r A_{n-1}\right] \mathrm{d} r=-\frac{a_{n}}{2},
$$

and thus $\alpha_{n-1}=-a_{n} /\left(2 a_{n-1}\right)$.

The new polynomials $A_{2}$ and $A_{4}$, weighted by their natural weight $\exp \left(-r^{2} / 2\right)$, are shown in Fig. 1 . Straightforward manipulations give also

$$
A_{2 n}^{\prime \prime}-r A_{2 n}^{\prime}+2 n A_{2 n}=4(n-1)(2 n+1) A_{2 n-2},
$$

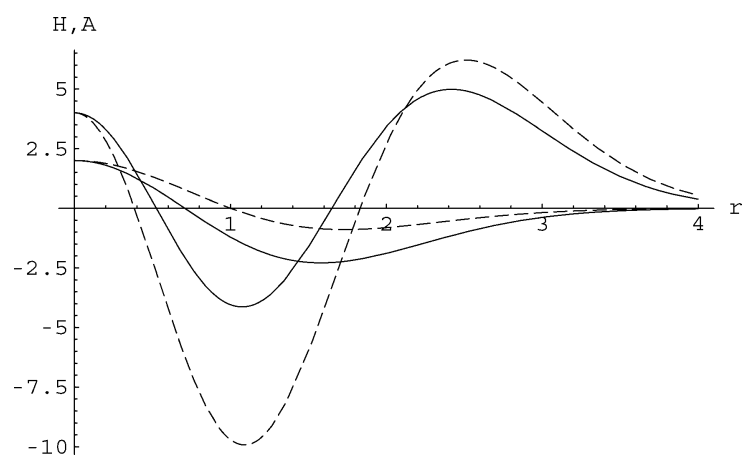

Fig. 1. Comparison of the Hermite polynomials $H_{2}$ and $H_{4}$ with the new polynomials $A_{2}$ and $A_{4}$. Full lines, $H_{2}(r)$ and $H_{4}(r)$, multiplied by $\exp \left(-r^{2} / 2\right)$ and normalized to 2 and 4 at the origin, respectively. Dashed lines, $A_{2}(r) \exp \left(-r^{2} / 2\right)$ and $A_{4}(r) \exp \left(-r^{2} / 2\right)$, also renormalized at the origin to 2 and 4 , respectively. 
and

$$
A_{2 n+1}^{\prime \prime}(r)-r A_{2 n+1}^{\prime}+(2 n+1) A_{2 n+1}=4 n(2 n+1) A_{2 n-1},
$$

or

$$
A_{n}^{\prime \prime}-r A_{n}^{\prime}+n A_{n}=2\left[n+(-1)^{n}\right]\left[n-1-(-1)^{n}\right] A_{n-2}
$$

Here, in the same way as a prime means first derivative, we used double primes for second derivatives. Furthermore, odd polynomials being Hermite polynomials, they satisfy the differential equation

$$
A_{2 n+1}^{\prime \prime}(r)-2 r A_{2 n+1}^{\prime}(r)+2(2 n+1) A_{2 n+1}(r)=0 .
$$

\section{Hermite polynomials constrained by a zero momentum}

Let the polynomials $B_{n}(r)$ of precise degree $n, n \neq 1$, be defined by

$$
\int_{-\infty}^{\infty} e^{-r^{2}} B_{m}(r) B_{n}(r) \mathrm{d} r=b_{n} \delta_{m n}, \quad m \neq 1, n \neq 1, \quad \int_{-\infty}^{\infty} e^{-\frac{1}{2} r^{2}} r B_{m}(r) \mathrm{d} r=0, \quad m \neq 1,
$$

where the $b_{n}$ are constants. The polynomial $B_{1}(r)$ is left out.

From the parity argument one sees that the even (indexed) polynomials are proportional to Hermite polynomials. They are not disturbed by leaving out $B_{1}(r)$ and only the odd (indexed) polynomials must be modified. In fact with $H_{n}(r)$ the usual Hermite polynomials, one can verify that

$$
B_{2 n+1}(r)=H_{2 n+1}(r)-3(n-1) ! 2^{2 n-1} \sum_{j=0}^{n-1} \frac{H_{2 j+1}(r)}{2^{2 j} j !}, \quad B_{2 n}(r)=H_{2 n}(r),
$$

satisfy Eqs. (17) for $m \neq 1, n \neq 1$, with

$$
b_{2 n+1}=2^{2 n+1}(2 n+1) !\left(1+\frac{3}{2 n}\right) \sqrt{\pi}, \quad b_{2 n}=2^{2 n}(2 n) ! \sqrt{\pi} .
$$

For this verification one needs Eqs. (5) and

$$
\int_{-\infty}^{\infty} e^{-\frac{1}{2} r^{2}} r H_{2 n+1}(r) \mathrm{d} r=\frac{(2 n+1) !}{n !} 2 \sqrt{2 \pi}
$$

with

$$
\sum_{j=0}^{n} \frac{(2 j+1) !}{2^{2 j+1}(j !)^{2}}=\frac{2}{3} \frac{(2 n+3) !}{2^{2 n+3} n !(n+1) !},
$$

which is a special case of Eq. (6) for $a=3 / 2$.

\section{Illustration: eigenmodes of density and potential perturbations}

Consider a one-dimensional toy model of $N$ non-interacting fermions embedded in a harmonic oscillator, with Hamiltonian $\mathcal{H}=\frac{1}{2} \sum_{i=1}^{N}\left(-\mathrm{d}^{2} / \mathrm{d} r_{i}^{2}+r_{i}^{2}\right)$. The ground state density is trivially obtained from the $N$ lowest orbitals, $\rho(r)=\sum_{i=1}^{N}\left[\psi_{i}(r)\right]^{2}$, where, trivially again, $\psi_{i}$ refers to the Hermite polynomial $H_{i-1}$, weighted by $\exp \left(-r^{2} / 2\right)$ and suitably square normalized to 1 . Assume now that a perturbation $\delta u$ is added to the harmonic potential. Let $i=1, \ldots, N$ and $I=N+1, N+2, \ldots, \infty$ label 'hole' and 'particle' orbitals, respectively. A standard argument provides the perturbative variation of the density,

$$
\delta \rho(r)=2 \sum_{i I} \psi_{i}(r) \psi_{I}(r) \frac{\langle I|\delta u| i\rangle}{\eta_{i}-\eta_{I}} .
$$

This reads, when $\delta u$ and $\delta \rho$ are expanded in that orthonormal basis $\{w\}$ provided by polynomials $A$,

$$
\delta \rho_{\beta}=2 \sum_{i I \alpha} \mathcal{D}_{\beta i I} \frac{\left\langle I\left|w_{\alpha}\right| i\right\rangle}{\eta_{i}-\eta_{I}} \delta u_{\alpha},
$$




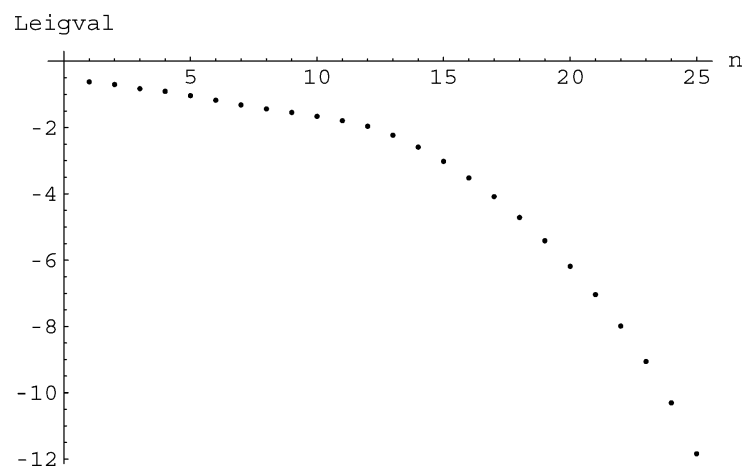

Fig. 2. Spectrum of $-\mathcal{F}$, in a $\log _{10}$ plot.

where $\mathcal{D}$ denotes the projection of a particle hole product of orbitals upon the basis $\left\{w_{\alpha}\right\}$,

$$
\mathcal{D}_{\beta i I}=\int \mathrm{d} r w_{\beta}(r) \psi_{i}(r) \psi_{I}(r) .
$$

The self energies $\eta$ for this toy model are, trivially, $\eta_{i}=i-1 / 2$ and $\eta_{I}=I-1 / 2$, but Eqs. (22)-(24) can be generalized for any mean field theory, naturally. Notice now that the perturbation matrix element $\left\langle I\left|w_{\alpha}\right| i\right\rangle$ is also a $\mathcal{D}$ matrix element, $\left\langle I\left|w_{\alpha}\right| i\right\rangle=\mathcal{D}_{\alpha i I}$. This exhibits the symmetric matrix

$$
\mathcal{F}=\mathcal{D} \frac{1}{E_{0}-\mathcal{H}} \tilde{\mathcal{D}}
$$

where $E_{0}-\mathcal{H}$ is a short notation to account for the energy denominators and the tilde indicates transposition. We shall call $\mathcal{F}$ a 'flexibility' matrix, as it connects potential perturbations and density perturbations. The symmetrical way in which this connection occurs seems remarkable.

In the following numerical example, where $N=4$, we slightly modified the basis $\{w\}$; rather than polynomials $A_{n}(r)$ we used polynomials $G_{n}(r) \propto A_{n}(\sqrt{2} r)$, with a suitable normalization, $\int_{-\infty}^{\infty} \mathrm{d} r \exp \left(-2 r^{2}\right) \sqrt{2 / \pi} G_{m}(r) G_{n}(r)=\delta_{m n}$. This is because products $\psi_{i} \psi_{I}$ carry a factor $\exp \left(-r^{2}\right)$, and we found it more reasonable to have the same exponential for $w_{\alpha}$ when calculating any overlap $\mathcal{D}_{\alpha i}$. We expect such a 'tail matching' to ensure a better convergence when summations $\sum_{i I}$ must be truncated, since the particle index $I$ cannot run to $\infty$ in practice. Thus we set $I \leqslant 101$, then considered even particle-hole states only, with a rule $i+I=$ even, and $\mathcal{F}$ was reduced to its positive parity submatrix with dimension 25 , namely the degree of $G_{n}$ ran from to 2 to 50 . Keeping in mind that $\mathcal{F}$ is semi-negative definite we show in Fig. 2 the $\log _{10}$ plot of its spectrum.

Let $X_{\gamma}^{m}$ denote the components of the $m$ th eigenvector of $\mathcal{F}$. Starting from the most negative eigenvalue, we show in Fig. 3 , together with the density $\rho(r)$, the first three 'density-potential' eigenmodes,

$$
D P_{m}(r)=\sum_{\gamma=1}^{25} X_{\gamma}^{m} w_{2 \gamma}(r)=(2 / \pi)^{\frac{1}{4}} \exp \left(-r^{2}\right) \sum_{\gamma=1}^{25} X_{\gamma}^{m} G_{2 \gamma}(r), \quad m=1,2,3 .
$$

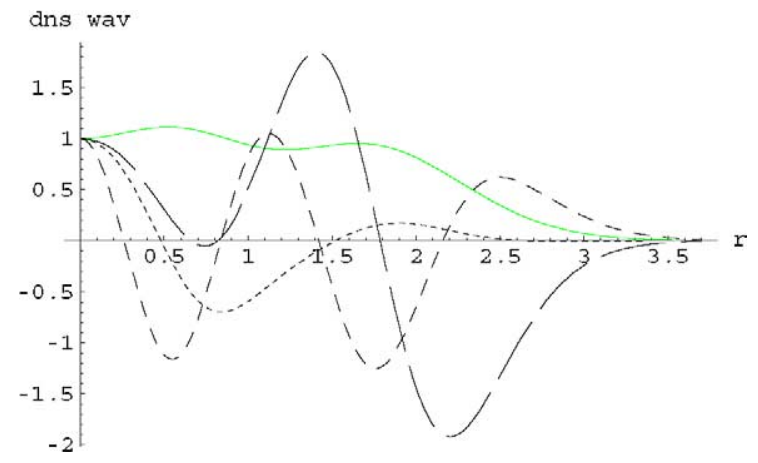

Fig. 3. Full line (thin): density generated by the first 4 Hermite polynomials. Very long dashes: lowest eigenmode generated by the 'flexibility' matrix $\mathcal{F}$. Dashed line: second mode. Dotted line: third mode. All curves are normalized to 1 at the origin. 
All curves are normalized to the same value 1 for $r=0$; for instance the true density is recovered with a factor $3 /(2 \sqrt{\pi})$. But it must stressed that the eigenmodes are infinitesimals, representing modulations of both the harmonic potential $r^{2} / 2$ and the true density, and that the ratios of such modulations are the eigenvalues of $\mathcal{F}$.

\section{Discussion and conclusion}

Our choice of the weight $e^{-r^{2} / 2}$ and of manipulations of Hermite polynomials was guided by nuclear physics, where the harmonic oscillator is not a bad first approximation to a mean field potential for the nuclear shell model. Simultaneously, we found a description of the 'tangent hyperplane' of the Hohenberg-Kohn functional. For atomic and molecular physics, however, a weight $e^{-r}$, because of Coulomb tails of orbitals, and manipulations of Laguerre polynomials are likely to be more relevant, obviously. Generalizations to three-dimensional problems are also in order.

A thorough investigation of numerical convergence when the particle and the 'new basis' labels are truncated is also necessary. Short range potentials, with a continuum for part of their spectrum, are more realistic than the harmonic potential, and will be a priority for a proper handling of Eqs. (22)-(24) when discrete sums turn into integrals.

It is clear that the present illustrative results are somewhat limited and that the number of similar problems, with other weights for orthogonality rules, is very large. Furthermore, the number of constraints is arbitrary, and any number of combinations of moments can be considered, in order to create other sets of polynomials.

We have not found yet a physical motivation for similar problems with Legendre polynomials, because physical constraints in angular space are not familiar to us. But these are not forgotten in our agenda.

The zoology of the 'flexibility' matrix with its eigenmodes already seems to make an intriguing problem. Furthermore our approach can clearly be generalized to mean fields of interacting particles. For want of a direct construction of the HohenbergKohn functional, information on its functional derivative is a by-product of the present approach.

\section{Appendix A}

Here are the first ten polynomials for the $A$ set,

$$
\begin{aligned}
& A_{1}(r)=2^{1}(r), \\
& A_{2}(r)=2^{2}\left(r^{2}-1\right), \\
& A_{3}(r)=2^{2}\left(2 r^{3}-3 r\right), \\
& A_{4}(r)=2^{3}\left(2 r^{4}-7 r^{2}+1\right), \\
& A_{5}(r)=2^{3}\left(4 r^{5}-20 r^{3}+15 r\right), \\
& A_{6}(r)=2^{4}\left(4 r^{6}-32 r^{4}+47 r^{2}-11\right), \\
& A_{7}(r)=2^{4}\left(8 r^{7}-84 r^{5}+210 r^{3}-105 r\right), \\
& A_{8}(r)=2^{5}\left(8 r^{8}-116 r^{6}+438 r^{4}-453 r^{2}+39\right), \\
& A_{9}(r)=2^{5}\left(16 r^{9}-288 r^{7}+1512 r^{5}-2520 r^{3}+945 r\right), \\
& A_{10}(r)=2^{6}\left(16 r^{10}-368 r^{8}+2600 r^{6}-6576 r^{4}+4881 r^{2}-633\right) .
\end{aligned}
$$

Here are the first ten polynomials for the $B$ set,

$$
\begin{aligned}
& B_{0}(r)=1, \\
& B_{2}(r)=2^{1}\left(2 r^{2}-1\right), \\
& B_{3}(r)=2^{3}\left(r^{3}-3 r\right), \\
& B_{4}(r)=2^{2}\left(4 r^{4}-12 r^{2}+3\right), \\
& B_{5}(r)=2^{4}\left(2 r^{5}-13 r^{3}+9 r\right), \\
& B_{6}(r)=2^{3}\left(8 r^{6}-60 r^{4}+90 r^{2}-15\right), \\
& B_{7}(r)=2^{5}\left(4 r^{7}-48 r^{5}+123 r^{3}-69 r\right), \\
& B_{8}(r)=2^{4}\left(16 r^{8}-224 r^{6}+840 r^{4}-840 r^{2}+105\right),
\end{aligned}
$$




$$
\begin{aligned}
& B_{9}(r)=2^{6}\left(8 r^{9}-156 r^{7}+846 r^{5}-1467 r^{3}+531 r\right), \\
& B_{10}(r)=2^{5}\left(32 r^{10}-720 r^{8}+5040 r^{6}-12600 r^{4}+9450 r^{2}-945\right) .
\end{aligned}
$$

\section{Acknowledgements}

It is a pleasure to thank B. Eynard, J.M. Luck, P. Moussa, J.M. Normand and L. Wilets for stimulating discussions about this problem.

\section{References}

[1] P. Hohenberg, W. Kohn, Phys. Rev. B 136 (3) (1964) 864.

[2] W. van Assche, Some applications of multiple orthogonal polynomials, in: Conference on Orthogonal Polynomials, Banff, Canada, March, 2004.

[3] N.D. Mermin, Phys. Rev. A 137 (5) (1965) 1441.

[4] R. Berg, L. Wilets, P. Phys. Soc. A LXVIII (1955) 229;

W. Kohn, L.J. Sham, Phys. Rev. A 140 (4) (1965) 1133. 